\title{
Commentary
}

\section{Overcoming the limitations of Rubisco; fantasy or realistic prospect?}

Douglas J. Orr, Martin A. J. Parry.

Lancaster Environment Centre, Lancaster University, Library Avenue, Lancaster, LA1 4YQ, UK.

Roughly 50 years since the discovery that the carbon-fixing enzyme Rubisco was also an oxygenase (Bowes et al., 1971), in this issue von Caemmerer reviews why this dual nature of Rubisco is key to both understanding and improving photosynthetic $\mathrm{CO}_{2}$ assimilation (von Caemmerer 2020). It highlights the pervasive role that Rubisco plays in shaping the atmosphere and biosphere, and how its biochemistry is integral to modelling photosynthetic gas exchange from individual leaves all the way to global scale. Tracing the trail of findings which ultimately led to the realisation that Rubisco was bifunctional, with $\mathrm{CO}_{2}$ and $\mathrm{O}_{2}$ acting as competitive inhibitors, provides the backdrop to how Rubisco biochemical properties were initially linked to leaf level measurements of gas exchange (see also the historical perspective on the oxygenase discovery by Bill Ogren himself in Ogren 2003).

As detailed by von Caemmerer (2020), the increased understanding of Rubisco biochemistry, and in particular the concentration of both the enzyme and its substrate RuBP within the chloroplast stroma, helped answer some of the questions previously raised through in vivo experimentation and observation. This allowed for the development of comprehensive models of photosynthesis, including the Farquhar-von Caemmerer-Berry model (FvCB, Farquhar et al., 1980). With the addition of new knowledge that has allowed for refinements, this model continues to be a pillar of photosynthesis research some four decades after the original publication, and established photosynthetic responses to $\mathrm{CO}_{2}$ - or A-Ci curves - as one of the most used methods in plant physiology.

The critical role of Rubisco, not only for parametrisation of scalable models of photosynthesis, but as a limitation on photosynthesis in many environmental settings, has made it a research focus. With advances in sequencing and technologies for in vitro analysis of Rubisco, this field is poised to continue growing in the near future. The review lays out many of the areas and topics currently targeted for increasing crop productivity via engineering. We explore these further, highlighting very recentlypublished work, and research on the dual nature of Rubisco as an oxygenase.

After the discovery of the oxygenase reaction of Rubisco, knowledge of sugar-phosphate derivatives that inhibited activity led to unsuccessful attempts to identify selective inhibitors of oxygenation (Keys et al., 1982). Given the current understanding of the structure of the enzyme, and that both reactions share initial steps, this is not surprising. A large body of work has investigated Rubisco catalytic properties from a wide range of sources and attempted to manipulate these properties via mutagenesis (von Caemmerer 2020). This work, alongside structural information from crystallography, greatly enhanced our understanding of the structure-function relationships between the large and small Rubisco subunits and the role of specific residues. However, it has otherwise met with little success because improvements in one kinetic parameter have often come at the expense of others, frequently involving specificity for $\mathrm{CO}_{2}$ vs $\mathrm{O}_{2}$, and thus the propensity for increased oxygenation. 
Although eliminating the oxygenase activity of Rubisco appears improbable, and indeed may be detrimental to the plant, there is demonstrated ample variation in plant Rubisco catalytic properties including specificity. Together with increased sequence information, this informs targeted attempts to exploit such variation. The recent development of the ability to express plant Rubisco in E. coli provides a substantial advance to the ability to rapidly test putative plant Rubisco improvements (Aigner et al., 2018). However, as noted by von Caemmerer, even with advances in tools to study the effect of manipulating Rubisco in planta, chloroplast transformation remains an obstacle to Rubisco large subunit engineering in major crops. The path toward exploiting the role of the small subunits by engineering appears clearer, with both rapid advances in nuclear gene editing technology, and an increasing appreciation of the importance of the small subunit in catalysis (e.g. Martin-Avila et al., 2020, Matsumara et al., 2020). Further variation in catalysis is evident from both extant forms of Rubisco, such as those identified through (wide) screening of diatoms and prokaryotes (Young et al., 2016, Davidi et al., 2020), and from analysis of Rubiscos generated via prediction or metagenomics tools (e.g. Shih et al., 2016, Banda et al., 2020).

The observed diversity in oxygenase activity and specificity in extant Rubisco forms means that it may be possible to manipulate this $\mathrm{CO}_{2} / \mathrm{O}_{2}$ selectivity in plants, a key component of Rubisco's impact on photosynthetic gas exchange. Some non-plant forms of Rubisco also appear more amenable to directed evolution approaches (e.g. Wilson et al., 2017), although reduced Rubisco amount as a result of biogenesis issues mean that even these fail to provide a suitable replacement for current crop Rubisco at this stage. Similarly, in the absence of an accompanying carbon concentrating mechanism (CCM), tobacco plants expressing cyanobacterial Rubisco with fast rates of carboxylation and low specificity require high $\mathrm{CO}_{2}$ to survive (Lin et al., 2014). A carboxysome- or pyrenoid-based carbon concentrating mechanism within the chloroplast would enable plants to operate with decreased amounts of Rubisco and increase nitrogen use efficiency, as Rubisco can account for well in excess of $20 \%$ of leaf nitrogen (von Caemmerer 2020). Introducing a cyanobacterial, algal or plant ( $C_{4}$ or CAM) $\mathrm{CCM}$ into major $\mathrm{C}_{3}$ crops such as rice is one of the most ambitious strategies to circumvent plant Rubisco deficiencies. The needs of engineering efforts continue to inspire, and necessitate, increased fundamental understanding of photosynthetic diversity.

Reducing the cost of Rubisco oxygenase activity through engineered photorespiratory bypasses may become especially relevant as the climate continues to warm, since high temperatures lead to reduced Rubisco specificity, increased frequency of oxygenation reactions, and hence photorespiration. Reviewed in detail by von Caemmerer (2020), the role of Rubisco activase in maintaining Rubisco activity, particularly during photosynthetic induction or high temperatures, is of continued interest. Faster Rubisco catalytic rates at high temperature promote the production of inhibitors via misfire reactions and make the role of Rubisco activase even more critical in maintaining Rubisco and photosynthetic activity in crops experiencing warmer temperatures.

Significant progress has been made in recent years in characterising Rubisco activase isoforms differing in thermal tolerance as well as better understanding factors limiting the speed of photosynthetic induction. This includes development of specific gas exchange protocols for making dynamic measurements during transitions from low to high light (e.g. Taylor et al., 2020). Increasing evidence of the potential gains in carbon assimilation to be made from accelerating the activation of Rubisco by Rubisco activase during photosynthetic induction make this an area of research ripe for exploring.

As emphasised by von Caemmerer, the central role of Rubisco in photosynthesis keeps the enzyme at the forefront of strategies aimed at increasing photosynthetic efficiency to improve crop yield and global food security, and to adapt agriculture to a changing climate. Despite limited success to date, 
improving Rubisco appears tantalisingly close, with the caveat that some significant hurdles remain. Encouragement can be drawn from a number of very recent advances in the ability to manipulate plant Rubisco both in vitro and in planta, and from continuing discoveries of diversity in form and capability of this critically important, massively abundant, yet imperfect enzyme.

\section{References cited.}

Aigner H, Wilson RH, Bracher A, Calisse L, Bhat JY, Hartl FU, Hayer-Hartl M (2018). Plant RuBisCo assembly in E. coli with five chloroplast chaperones including BSD2. Science. 358, 1272-1278

Banda DM, Pereira JH, Liu AK, Orr DJ, Hammel M, He C, Parry MAJ, Carmo-Silva E, Adams PD, Banfield JF. Shih PM (2020). Novel Bacterial Clade Reveals Origins of Form I Rubisco. Nature Plants. DOI: 10.1038/s41477-020-00762-4.

Bowes G, Ogren WL, Hageman RH (1971). Phosphoglycolate production catalyzed by ribulose diphosphate carboxylase. Biochemical and Biophysical Research Communications. 45, 716-722.

Davidi D, Shamshoum M, Guo Z, Bar-On YM, Prywes N, Oz A, Jablonska J, Flamholz A, Wernick DG, Antonovsky N, de Pins B, Shachar L, Hochhauser D, Peleg Y, Albeck S, Sharon I, Mueller-Cajar O, Milo $R$ (2020). Highly active rubiscos discovered by systematic interrogation of natural sequence diversity. The EMBO Journal, e104081.

Farquhar GD, von Caemmerer S, Berry JA (1980). A biochemical-model of photosynthetic $\mathrm{CO}_{2}$ assimilation in leaves of $C_{3}$ species. Planta. 149, 78-90.

Keys AJ, Bird IF, Cornelius MJ (1982). Possible use of chemicals for the control of photorespiration. In: McLaren JS (eds) Chemical manipulation of crop growth and development. Butterworths, London, UK.

Lin M, Occhialini A, Andralojc P, Parry MAJ, Hansom MR (2014). A faster Rubisco with potential to increase photosynthesis in crops. Nature 513, 547-550.

Martin-Avila E, Lim Y, Birch R, Dirk LMA, Buck S, Rhodes T, Sharwood RE, Kapralov MV, Whitney SM (2020). Modifying plant photosynthesis and growth via simultaneous chloroplast transformation of Rubisco large and small subunits. The Plant Cell. 32, 2898-2916.

Matsumura H, Shiomi K, Yamamoto A, Taketani Y, Kobayashi N, Yoshizawa T, Tanaka S-i, Yoshikawa H, Endo M, Fukayama H (2020). Hybrid Rubisco with Complete Replacement of Rice Rubisco Small Subunits by Sorghum Counterparts Confers C4-Plant-like High Catalytic Activity. Molecular Plant. DOI: 10.1016/j.molp.2020.08.012

Ogren WL (2003) Affixing the $O$ to Rubisco: discovering the source of photorespiratory glycolate and its regulation. Photosynthesis Research. 76, 53-63.

Shih PM, Occhialini A, Cameron JC, Andralojc PJ, Parry MAJ, Kerfeld CA (2016). Biochemical characterization of predicted Precambrian RuBisCO. Nature Communications. 7, 10382.

Taylor SH, Orr DJ, Carmo-Silva E, Long SP. (2020) During photosynthetic induction, biochemical and stomatal limitations differ between Brassica crops. Plant, Cell \& Environment. DOI: 10.1111/pce.13862. 
Von Caemmerer S (2020) Rubisco carboxylase/oxygenase: From the enzyme to the globe: A gas exchange perspective. Journal of Plant Physiology. 252, 153240.

Wilson RH, Martin-Avila E, Conlan C, Whitney SM (2017). An improved Escherichia coli screen for Rubisco identifies a protein-protein interface that can enhance $\mathrm{CO}$-fixation kinetics. Journal of Biological Chemistry. 293, 18-27

Young JN, Heureux AMC, Sharwood RE, Rickaby REM, Morel FMM, Whitney SM (2016). Large variation in the Rubisco kinetics of diatoms reveals diversity among their carbon-concentrating mechanisms. Journal of Experimental Botany. 67, 3445-3456. 